\title{
Genetic and Environmental Influences on Pulmonary Function and Muscle Strength: The Chinese Twin Study of Aging
}

\author{
Xiaocao Tian, ${ }^{1,2}$ Chunsheng $\mathrm{Xu}^{2,3}{ }^{2}$ Yili Wu$^{3}$ Jianping Sun, ${ }^{2,3}$ Haiping Duan, ${ }^{2,3}$ Dongfeng Zhang, ${ }^{3}$ \\ Baofa Jiang, ${ }^{1}$ Zengchang Pang, ${ }^{2}$ Shuxia $\mathrm{Li}^{4}{ }^{4}$ and Qihua $\mathrm{Tan}^{4,5}$ \\ ${ }^{1}$ Department of Epidemiology, School of Public Health, Shandong University, Jinan, Shandong, China \\ ${ }^{2}$ Qingdao Municipal Center for Disease Control and Prevention, Qingdao University Medical College, Qingdao, \\ Shandong, China \\ ${ }^{3}$ Department of Epidemiology and Health Statistics, Qingdao University Medical College, Qingdao, Shandong, China \\ ${ }^{4}$ Department of Clinical Research, Unit of Human Genetics, University of Southern Denmark, Odense, Denmark \\ ${ }^{5}$ Department of Public Health, Epidemiology, Biostatistics and Biodemography, University of Southern Denmark, Odense, \\ Denmark
}

\begin{abstract}
Genetic and environmental influences on predictors of decline in daily functioning, including forced expiratory volume in $1 \mathrm{~s}\left(\mathrm{FEV}_{1}\right)$, forced vital capacity (FVC), handgrip, and five-times-sit-to-stand test (FTSST), have not been addressed in the aging Chinese population. We performed classical twin modeling on $\mathrm{FEV}_{1}$, FVC, handgrip, and FTSST in 379 twin pairs (240 MZ and 139 DZ) with median age of 50 years (40-80 years). Data were analyzed by fitting univariate and bivariate twin models to estimate the genetic and environmental influences on these measures of physical function. Heritability was moderate for FEV 1 , handgrip, and FTSST (55-60\%) but insignificant for FVC. Only FVC showed moderate control, with shared environmental factors accounting for about $50 \%$ of the total variance. In contrast, all measures of pulmonary function and muscle strength showed modest influences from the unique environment (40-50\%). Bivariate analysis showed highly positive genetic correlations between $\mathrm{FEV}_{1}$ and $\mathrm{FVC}\left(r_{\mathrm{G}}=1.00\right)$, and moderately negative genetic correlations between FTSST and FEV ${ }_{1}\left(r_{\mathrm{G}}=-0.33\right)$ and FVC $\left(r_{\mathrm{G}}=-0.42\right) . \mathrm{FEV}_{1}$ and FVC, as well as $\mathrm{FEV}_{1}$ and handgrip, displayed high common environmental correlations $\left(r_{C}=1.00\right)$, and there were moderate correlations between FVC and handgrip $\left(r_{C}=0.44\right)$. $\mathrm{FEV}_{1}$ and $\mathrm{FVC}$ showed high unique environmental correlations $\left(r_{\mathrm{E}}=0.76\right)$ and low correlations between handgrip and FEV ${ }_{1}\left(r_{\mathrm{E}}=0.17\right), \mathrm{FVC}\left(r_{\mathrm{E}}=0.14\right)$, and FTSST ( $r_{E}=-0.13$ ) with positive or negative direction. We conclude that genetic factors contribute significantly to the individual differences in common indicators of daily functioning (FEV 1 , handgrip, and FTSST). $\mathrm{FEV}_{1}$ and FVC were genetically and environmentally correlated. Pulmonary function and FTSST may share similar sets of genes but in the negative direction. Pulmonary function and muscle strength may have a shared environmental background.
\end{abstract}

Keywords: pulmonary function, muscle strength, heritability, genetic correlation, Chinese twins

The elderly in the Chinese population has increased remarkably in the past three decades, a blessing that comes with an increased public health burden as aging coincides with an increase in comorbid chronic disorders. With the unprecedented increase in average life expectancy, researchers have sought to establish the mechanism of healthy aging, with special attention given to traits that contribute to quality of life, such as pulmonary function and muscle strength. Forced expiratory volume in $1 \mathrm{~s}\left(\mathrm{FEV}_{1}\right)$, forced vital capacity (FVC), handgrip, and five-times-sitto-stand test (FTSST) are inexpensive, easily measurable, and preferred indicators of pulmonary function and mus- cle strength. $\mathrm{FEV}_{1}$, a trait that measures airflow, and FVC, a trait that measures lung volume, are the two most important and standard indicators of pulmonary function.

RECEIVED 1 August 2016; ACCEPTED 26 September 2016

ADDRESS FOR CORRESPONDENCE: Professor Dongfeng Zhang, Department of Public Health, Qingdao University Medical College, Qingdao, Shandong, China. E-mail: zhangdf1961@126.com and Baofa Jiang, Department of Epidemiology, School of Public Health, Shandong University, Jinan, Shandong, China. E-mail: bjiang@sdu.edu.cn 
Handgrip and FTSST are representatives of skeletal muscle strength, which is known to be associated with muscular functioning in other muscle groups and with activities of daily living (ADL). A person's ability or inability to perform ADLs is the most used measure of functional status, particularly in people with chronic disabilities and the elderly (Rantanen et al., 2002).

Aging is accompanied by a progressive decline in $\mathrm{FEV}_{1}$, FVC, and handgrip, and an increase in FTSST. Declining pulmonary function and muscle strength late in life is a major problem that severely hampers independent living and thus quality of life. Previous studies have shown that poor pulmonary function and muscle strength (Rantanen et al., 2002) is a risk for disability, morbidity, and mortality in elderly people.

In the past decades, the classical twin method has been applied to estimate the genetic and environmental contributions to phenotypes of pulmonary function and muscle strength in Western countries, including $\mathrm{FEV}_{1}, \mathrm{FVC}$, handgrip, and FTSST. The heritability estimates of $\mathrm{FEV}_{1}$ and FVC were 32-67\% and 26-56\% (Ghio et al., 1989; Givelber et al., 1998; Hukkinen et al., 2011; Ingebrigtsen et al., 2011; Klimentidis et al., 2013; McClearn et al., 1994; Redline et al., 1989; Wilk et al., 2000; Xu et al., 1999), respectively in adult twins, with environmental factors explaining a modest part of the variance in most of the studies.

Genetic factors accounted for $57 \%$ of the variance in repeated chair stands performance of the lower extremity strength among elderly male twins (Carmelli et al., 2000 ) and for $22-52 \%$ of the total variance in handgrip strength (Arden \& Spector, 1997; Carmelli \& Reed, 2000; Frederiksen et al., 2002; Reed et al., 1991). However, a multivariate genetic analysis showed a much more modest additive genetic effect (14\%) in older female twins aged 63-76 years (Tiainen et al., 2004). Univariate analyses in this study even suggested that the CE model showed the best fit for handgrip strength, suggesting no genetic effect on handgrip.

In contrast to Western countries, far less is known about the genetic and environmental influences on aging-related indicators of pulmonary function and muscle strength in the rapidly aging Chinese population. This article reports results from the first wave of data on pulmonary function and muscle strength in Chinese middle- and old-aged twins as part of a comprehensive investigation on genetic and environmental regulation on aging indices or traits.

\section{Methods}

\section{Study Participants}

Sample collection was conducted through the Qingdao Twin Registry (QTR), the first twin registry in China (Li et al., 2006; Li et al., 2013) established at Qingdao Center for Disease Control and Prevention (Qingdao CDC; Duan et al., 2013; Pang et al., 2006). We recruited the twin samples through QTR, local CDC, community, and media publicity from 2012 to 2013. Twins unable or unwilling to cooperate, suffering from pulmonary and skeletal muscle disease, professional athletes, and incomplete twin pairs were excluded from sampling. We included a total of 379 complete adult twin pairs, including 240 monozygotic (MZ) twins (114 MZ males [MZM], $126 \mathrm{MZ}$ females [MZF]) and 139 dizygotic (DZ) twins (41 DZ males [DZM], 39 DZ females [DZF], 59 opposite-sex DZ [OSDZ]) aged $51.8 \pm 7.6$ years $(40-$ 80 years old). The participants included came from all social classes and from different occupations, so they did not represent a specific socio-economic status. Zygosity was identified using DNA markers with $99.9 \%$ correct assignment (Becker et al., 1997; Tomsey et al., 2001) at a laboratory at the Qingdao Blood Center.

Institutional Review Boards of Qingdao CDC approved the study. We conducted the study in accordance with the ethical principles of the Helsinki Declaration. All volunteer twin participants gave the informed consent and then completed the questionnaire and physical examination at Qingdao CDC.

\section{Measurements of Physical Function}

$\mathrm{FEV}_{1}$ and FVC (liters) were measured by the electronic hand-held spirometer (Micro 0102) to assess pulmonary function. Every participant performed two maneuvers while standing, according to the standard procedure of spirometry. Duplicate tests were made and the best trial data were used for analysis. Handgrip strength was measured in kilograms by using an electronic dynamometer (WCS-100, Nantong, China). Participants were asked to squeeze the handle as hard as possible in a standing position with the arm straight down. Handgrip strength was assessed as the maximum of six attempts with three tests on each hand. The FTSST (seconds) was adopted to measure the lower limb strength. The participants were asked to fold their arms across the chest and rise from the chair and then sit down fully as fast as they could without pause. The time spent to complete the test was recorded using a stop watch.

The spirometer, dynamometer, and stop watch were calibrated every morning before measurement.

\section{Statistical Analysis}

Epidata3.1 (www.epidata.dk) was used to input and correct the data. Because the distributions of four variables were skewed, the Box-Cox transformation was applied to ensure normal or approximately normal distribution. The Box-Cox transformation was done using the free R package car (http://cran.r-project.org/web/packages/car/index. html). In the model fitting, age, sex, and age*sex were included as the covariates to adjust for their effects on pulmonary function and muscle strength. Because smokers and non-smokers did not differ significantly on pulmonary function, data were not corrected for smoking status (Finkel 
et al., 2013). Twin correlation was measured by calculating the intra-class coefficients (ICCs) and compared between MZ and DZ. ICCs were estimated using twinlm in the $\mathrm{R}$ package mets (http://cran.r-project.org/web/packages/ mets/index.html). Phenotypic correlation among the four indicators was assessed by cross-twin, cross-trait correlation (CTCTC) calculated using R package car (http://cran. r-project.org/web/packages/car/index.html).

\section{Twin Modeling}

We fitted classical univariate twin models to decompose the total variance of every indicator of pulmonary function and muscle strength into the components of additive genetic (A), common environmental (C), dominant genetic (D), and unique environmental (E) variances by constructing the full ACE model or the ADE model. The ADE model was only taken into account when the ICC of MZ twins was more than twice as large as that of DZ twins. The E term includes any measurement error and can therefore never be zero. Following the parsimony principle, nested models, such as AE, CE, DE, E were fitted by dropping the relevant variance components. Statistical significance of nested models was tested by a likelihood ratio test and we chose the best-fitting model based on the parsimony principle and the Akaike's Information Criterion (AIC; Akaike, 1987). In addition to the univariate twin modeling described above, we also fitted bivariate twin modeling to assess the genetic and environmental correlations between indicators of pulmonary function and muscle strength. The CTCTC was estimated before fitting the bivariate model and compared across zygosity to provide indication of genetic mediation between the two traits of interest. Twin models were fitted by R package mets for the univariate analysis and Mx (http: //www.vcu.edu/mx) for the bivariate analysis. The level of statistical significance was set at $p>.05$.

\section{Results}

In Table 1, we show the descriptive statistics together with results from fitting linear models to each measurement for the effects of age, sex, and age* sex. We found that age had a significant effect on the four phenotypes. There was a strong pattern of decline in $\mathrm{FEV}_{1}, \mathrm{FVC}$, and handgrip whereas FTSST increased with age, as indicated by the highly significant negative and positive regression coefficients, respectively. For sex, we found lower $\mathrm{FEV}_{1}, \mathrm{FVC}$, and handgrip in females than in males (males coded 1 and females 2). The FTSST did not differ between females and males. There were significant age-gender interactions in favor of females, meaning that females outperformed males in $\mathrm{FEV}_{1}$ and handgrip. Table 1 also has ICCs for all measurements for $\mathrm{MZ}$ and DZ twins estimated separately. Overall, MZ twins had significantly higher ICCs than that for DZ twins for all items $(p>.05)$ except FVC. This provided a first indication of genetic influences on $\mathrm{FEV}_{1}$, handgrip, and FTSST.

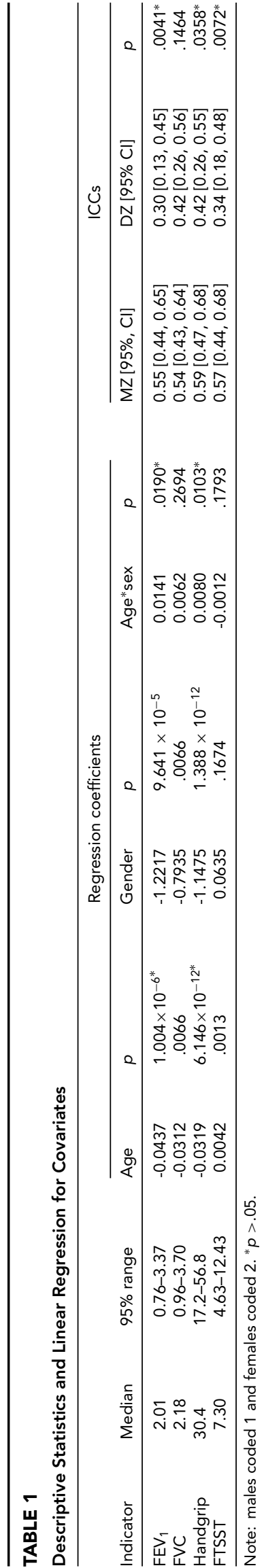


Because all the ICCs in MZ were less than twice those in $\mathrm{DZ}$, we fitted the full ACE and its nested models (AE, CE, E) to each of the four indicators of pulmonary function and muscle strength. We analyzed the variance components and heritability estimates after adjustment for all covariates. The most parsimonious model for $\mathrm{FEV}_{1}$, handgrip, and FTSST was the AE model, while FVC had the CE model as its bestfitting model (Table 2). There were significant additive genetic influences on $\mathrm{FEV}_{1}$, handgrip, and FTSST. In the AE models, moderate heritability (55-60\%) was estimated with 95\% CIs well beyond zero. In the CE model for FVC, the common environment contribution was about $50 \%$. All the four phenotypes appeared to be similar with regard to the magnitude of effects of the unique environmental factors (40-50\%).

We found that $\mathrm{FEV}_{1}, \mathrm{FVC}$, and handgrip had significant differences in CTCTC between MZ and DZ twins (Table 3). CTCTCs were higher in MZ than in DZ twins except between handgrip and FTSST, implying potential genetic involvement in the correlations of pulmonary function and muscle strength. So, we additionally fitted the bivariate twin models to estimate the genetic and environmental correlations for the indicators of pulmonary function with muscle strength. Similar to the univariate analysis, both full and nested models were fitted for best-fitting model selection, with the nested models fitted by dropping the genetic and environmental components in the covariance between every combination of these four indices. In Table 4, we show the estimated genetic and environmental correlations in the full and best-fitting models for the combinations of pulmonary function with muscle strength. The correlations between $\mathrm{FEV}_{1}$ and FVC were best fitted by the ACE model. $\mathrm{FEV}_{1}, \mathrm{FVC}$, as well as handgrip and FTSST had the AE model as the best-fitting model. $\mathrm{FEV}_{1}$, as well as FVC and handgrip, had the $\mathrm{CE}$ model as the best-fitting model. The genetic correlation between $\mathrm{FEV}_{1}$ and FVC was $r_{\mathrm{G}}=$ 1.00 [95\% CI: $0.87,1.00$ ], the moderately negative genetic correlation between $\mathrm{FEV}_{1}$ and FTSST was $r_{\mathrm{G}}=-0.33$, [95\% CI: $-0.68,-0.14$ ], between FVC and FTSST was $r_{\mathrm{G}}$ $=-0.42[95 \% \mathrm{CI}:-1.00,-0.13]$. We found no significant genetic correlation between handgrip and pulmonary function, or FTSST. FEV 1 and FVC also presented a high positive common environmental correlation, with $r_{\mathrm{C}}=$ 1.00 [95\% CI: 0.74, 1.00], and between $\mathrm{FEV}_{1}$ and handgrip, $r_{\mathrm{C}}=1.00$ [95\% CI: $\left.0.31,1.00\right]$, and moderately common environmental correlation between FVC and handgrip, with $r_{\mathrm{C}}=0.44$ [95\% CI: 0.17, 1.00]. Furthermore, FEV $\mathrm{F}_{1}$ and FVC showed high unique environmental correlation, with $r_{\mathrm{E}}=0.76$ [95\% CI: 0.71, 0.81], mild unique environmental correlation between handgrip and $\mathrm{FEV}_{1}, r_{\mathrm{E}}=0.17[95 \%$ CI: 0.06, 0.28], and FVC, $r_{\mathrm{E}}=0.14$ [95\% CI: 0.03, 0.25], as well as FTSST, $r_{\mathrm{E}}=-0.13$, [95\% CI: $-0.25,-0.01$, with positive or negative directions, respectively. The estimates on common and the unique environmental correlations for the other combinations were all zero.

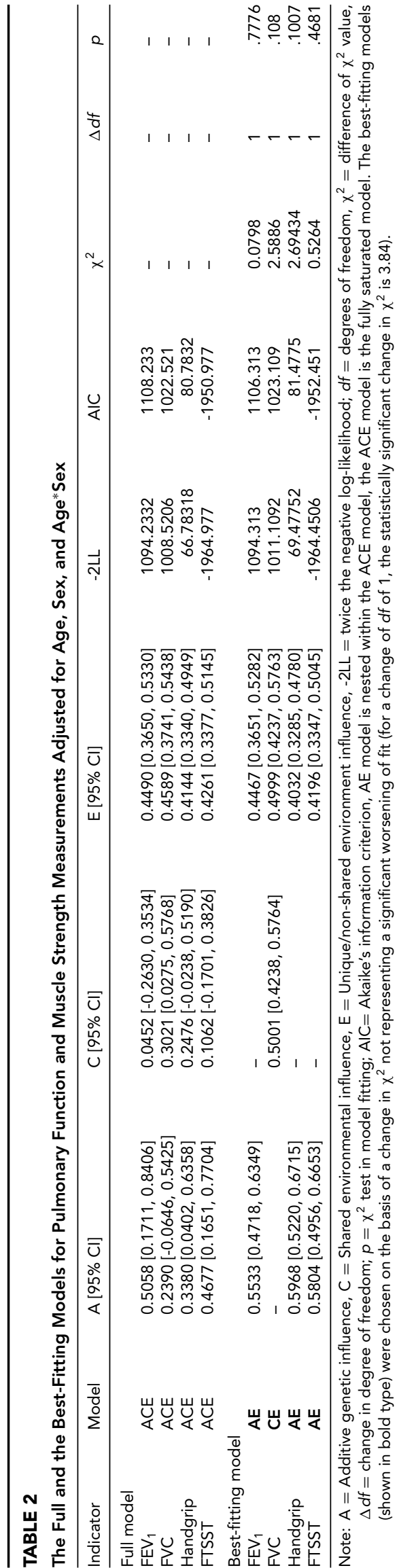




\section{TABLE 3}

Phenotypic Correlations (Cross-Twin, Cross-Trait Correlation) Between Pulmonary Function and Muscle Strength

\begin{tabular}{lccccc}
\hline Indicators pair & $\mathrm{MZ}$ & $\mathrm{n}_{\mathrm{MZ}}$ & \multicolumn{1}{c}{$\mathrm{DZ}$} & $\mathrm{n}_{\mathrm{DZ}}$ & \multicolumn{1}{c}{$\mathrm{p}$} \\
\hline FEV $_{1}$-FVC & 0.63 & 238 & 0.32 & 139 & .0001 \\
FEV $_{1}$-Handgrip & 0.46 & 232 & 0.08 & 137 & .0001 \\
FEV $_{1}$-FTSST & -0.20 & 235 & -0.08 & 136 & .2598 \\
FVC-Handgrip & 0.45 & 232 & 0.04 & 137 & $4.3356 \times 10^{-5}$ \\
FVC-FTSST & -0.16 & 235 & -0.04 & 136 & .2644 \\
Handgrip-FTSST & -0.02 & 229 & -0.02 & 131 & 1.000 \\
\hline
\end{tabular}

\section{Discussion}

We have conducted the first twin study on aging-related indicators of pulmonary function and muscle strength in a population-based sample of healthy adult Chinese twins. The current study demonstrated that there was strong evidence of genetic influence on $\mathrm{FEV}_{1}$ and indices of muscle strength in middle- and old-aged twins. Our analysis obtained a heritability of more than $50 \%$ for $\mathrm{FEV}_{1}$, handgrip, and FTSST. For FVC shared and unique environmental factors, both explained the same proportion of total variance of $50 \%$.

The results indicated that about $55 \%$ of the variance in the level of $\mathrm{FEV}_{1}$ in a middle- and old-aged Chinese population may be explained by genetic factors. However, no significantly genetic contribution of FVC was estimated. Pulmonary function has been found previously to have a heritable basis in Western countries. Our estimates of heritability were similar to previous studies (Klimentidis et al., 2013) but the estimate of genetic component of FVC was different from the 26\% found by Ingebritsen et al. (2011). The reason may lie in the different social, cultural, and environmental factors between China and Western countries or the relatively small sample size in the present study. Further investigation of genetic mechanisms with respect to pulmonary function is needed.

Our results indicated a similar heritability of handgrip strength as that of Frederiksen et al. (2002) of 52\% but higher than that of Arden and Spector (1997) of 30\%. It has been observed previously that the relative contributions of genetic and environmental effects to handgrip strength may change over the years. For example, in a study performed by Carmelli and Reed (2000), the heritability of handgrip strength decreased from $35 \%$ to $22 \%$ during 10 years follow-up, whereas the shared environmental component increased from $39 \%$ to $45 \%$ in male twins aged 63 years old on average at the baseline. We considered that the relatively high heritability of handgrip in the present study could be due to the relatively younger participants compared with those of Western studies. The current study estimated a genetic contribution to FTSST, which was comparable to $57 \%$ reported by Carmelli et al. (2000).

The same level of genetic (55-60\%) and unique environmental (40-45\%) contributions were found for $\mathrm{FEV}_{1}$, handgrip, and FTSST. Approximately equal contribution of genetic (58-60\%) and unique environmental factors (40$42 \%$ ) was estimated between handgrip and FTSST, the two indicators of muscle strength. We were surprised to see that the non-shared environmental effects on all four phenotypes of pulmonary function and muscle strength were also quite similar $(40-50 \%)$ in the univariate analysis of the present study. Environmental effects were primarily dominated by unique and stochastic effects rather than to common exposures of both twins. Measurement error would have inflated the relative contribution of the non-shared environmental effects. Only FVC presented a significant common and unique environmental influence, which exerted approximately equal impact on FVC according to the estimates. No role of the shared environment at all was found for $\mathrm{FEV}_{1}$, handgrip, and FTSST.

The genetic correlation expresses the extent to which two measurements of pulmonary function and muscle strength reflect the same set of genes. Study of the genetic correlation can facilitate exploration of pleiotropic genetic variants. The present study showed that $\mathrm{FEV}_{1}, \mathrm{FVC}$, and FTSST may share the same or similar sets of genes but with a positive or negative direction. The bivariate genetic analysis showed that the significantly genetic correlation between FTSST and FEV 1 as well as FVC was moderate but in the opposite direction, which was in line with the corresponding CTCTC estimate, suggesting that the pulmonary function and FTSST may share a similar genetic basis that moderately regulated the elevated $\mathrm{FEV}_{1}, \mathrm{FVC}$, and decreased FTSST at the same time and vice versa. As the decreased FTSST meant enhanced lower limb strength, we thought that the underlying genes may have positive pleiotropic effects on both pulmonary function and lower limb strength. We found no significant genetic correlation between handgrip and the three other indicators, implying handgrip may be dominated by its own genetic factors. Interestingly, we found that $\mathrm{FEV}_{1}, \mathrm{FVC}$, and handgrip may share the same or similar common environmental factors. In contrast to the genetic correlation, we observed that handgrip and the other three indicators presented mild unique environmental correlations, indicating that handgrip, $\mathrm{FEV}_{1}$, and FVC, as well as FTSST, may share similar unique environmental factors.

Improved knowledge of the genetic basis of pulmonary function and muscle strength could lead to a better understanding of the genetic architecture of these traits. Recent genome-wide association studies have identified several loci that are associated with pulmonary function and are biologically plausible candidates (Tang et al., 2014). A polymorphism of the IGF2 gene has been observed to have an effect on adult handgrip strength among men (Sayer et al., 2002). The results of the present study implied that pulmonary function and lower limb strength may be regulated by the same or a common set of genes. However, the specific genes regulating these 
TABLE 4

Estimated Genetic and Environmental Correlations Between Indicators

\begin{tabular}{|c|c|c|c|c|c|c|}
\hline \multirow[b]{2}{*}{ Indicator pair } & \multicolumn{3}{|c|}{ Full models } & \multicolumn{3}{|c|}{ Best-fitting models } \\
\hline & $r_{\mathrm{G}}[95 \% \mathrm{Cl}]$ & $r_{\mathrm{C}}[95 \% \mathrm{Cl}]$ & $r_{E}[95 \% \mathrm{Cl}]$ & $r_{\mathrm{G}}[95 \% \mathrm{Cl}]$ & $r_{\mathrm{C}}[95 \% \mathrm{Cl}]$ & $r_{\mathrm{E}}[95 \% \mathrm{Cl}]$ \\
\hline $\mathrm{FEV}_{1}-\mathrm{FVC}$ & $1.00[0.87,1.00]$ & $1.00[0.74,1.00]$ & $0.76[0.71,0.81]$ & $1.00[0.87,1.00]^{*}$ & $1.00[0.74,1.00]^{*}$ & $0.76[0.71,0.81]^{*}$ \\
\hline $\mathrm{FEV}_{1}-$ Handgrip & $0.01[-0.87,0.55]$ & $1.00[-1.00,1.00]$ & $0.17[0.05,0.29]$ & - & $1.00[0.31,1.00]^{*}$ & $0.17[0.06,0.28]^{*}$ \\
\hline $\mathrm{FEV}_{1}-\mathrm{FTSST}$ & $-0.40[-0.96,0.05]$ & $0.67[-1.00,1.00]$ & $0.04[-0.09,0.16]$ & $-0.33[-0.68,-0.14]^{*}$ & - & $0.03[-0.09,0.15]$ \\
\hline FVC-Handgrip & $0.09[-1.00,1.00]$ & $0.38[-0.54,1.00]$ & $0.13[0.01,0.25]$ & - & $0.44[0.17,1.00]^{*}$ & $0.14[0.03,0.25]^{*}$ \\
\hline FVC-FTSST & $-0.76[-1.00,-0.12]$ & $0.66[-1.00,1.00]$ & $0.05[-0.08,0.17]$ & $-0.42[-1.00,-0.13]^{*}$ & - & $0.03[-0.10,0.15]$ \\
\hline Handgrip-FTSST & $-0.09[-0.74,0.66]$ & $0.02[-1.00,1.00]$ & $-0.13[-0.25,0.01]$ & $-0.08[-0.40,0.21]$ & - & $-0.13[-0.25,-0.01]^{*}$ \\
\hline
\end{tabular}

Note: $r_{\mathrm{G}}=$ genetic correlation between traits; $r_{\mathrm{C}}=$ shared environmental correlation between traits; $r_{\mathrm{E}}=$ non-shared environmental correlation between traits. $* p>.05$.

indicators remained to be defined by molecular genetic studies.

A strength of our study was the evaluation of four representative aspects of aging-related pulmonary function and muscle strength at the same time. However, it is necessary to point out the limitations of our study. Compared with published studies from Western countries, the sample size was relatively small, which resulted in less reliable heritability estimates of phenotypes of pulmonary function and muscle strength. Another limitation is the relatively young age of our twin samples and the large span of age covered (40 years). Although age was adjusted in the twin modeling, the non-linear pattern, if any, of age-dependent change in pulmonary function and muscle strength could have been averaged out and could also serve to reduce power of the study.

\section{Acknowledgments}

This study was supported by National Natural Science Foundation of China (31371024). All the authors contributed significantly to the study. X.T., C.X., Y.W., J.S., H.D.: sample collection, conduct of the study, drafting the manuscript; D.Z., B.J., Z.P.; design of the study, data preparation; S.L., Q.T.: data analysis strategy, interpretation, manuscript revision.

\section{Conflict of Interest}

None.

\section{References}

Akaike, H. (1987). Factor analysis and AIC. Psychometrika, 52, $317-332$

Arden, N. K., \& Spector, T. D. (1997). Genetic influences on muscle strength, lean body mass, and bone mineral density: A twin study. Journal of Bone and Mineral Research, 12, 2076-2081.

Becker, A., Busjahn, A., Faulhaber, H. D., Bahring, S., Robertson, J., Schuster, H., \& Luft, F. C. (1997). Twin zygosity. Automated determination with microsatellites. Journal of Reproductive Medicine, 42, 260-266.
Carmelli, D., Kelly-Hayes, M., Wolf, P. A., Swan, G. E., Jack, L. M., Reed, T., \& Guralnik, J. M. (2000). The contribution of genetic influences to measures of lower-extremity function in older male twins. The Journal of Gerontology, Series A, Biological Sciences and Medical Sciences, 55, B49-B53.

Carmelli, D., \& Reed, T. (2000). Stability and change in genetic and environmental influences on hand-grip strength in older male twins. Journal of Appllied Physiology, 89, 18791883.

Duan, H., Ning, F., Zhang, D., Wang, S., Zhang, D., Tan, Q., ... Pang, Z. (2013). The qingdao twin registry: A status update. Twin Research and Human Genetics, 16, 79-85.

Finkel, D., Reynolds, C. A., Emery, C. F., \& Pedersen, N. L. (2013). Genetic and environmental variation in lung function drives subsequent variation in aging of fluid intelligence. Behavior Genetics, 43, 274-285.

Frederiksen, H., Gaist, D., Petersen, H. C., Hjelmborg, J., McGue, M., Vaupel, J. W., \& Christensen, K. (2002). Hand grip strength: A phenotype suitable for identifying genetic variants affecting mid- and late-life physical functioning. Genetic Epidemiology, 23, 110-122.

Ghio, A. J., Crapo, R. O., Elliott, C. G., Adams, T. D., Hunt, S. C., Jensen, R. L., ... Afman, G. H. (1989). Heritability estimates of pulmonary function. Chest, 96, 743-746.

Givelber, R. J., Couropmitree, N. N., Gottlieb, D. J., Evans, J. C., Levy, D., Myers, R. H., \& O’Connor, G. T. (1998). Segregation analysis of pulmonary function among families in the Framingham study. American Journal of Respiratory and Critical Care Medicine, 157, 1445-1451.

Hukkinen, M., Kaprio, J., Broms, U., Viljanen, A., Kotz, D., Rantanen, T., \& Korhonen, T. (2011). Heritability of lung function: A twin study among never-smoking elderly women. Twin Research and Human Genetics, 14, 401-407.

Ingebrigtsen, T. S., Thomsen, S. F., van der Sluis, S., Miller, M., Christensen, K., Sigsgaard, T., \& Backer, V. (2011). Genetic influences on pulmonary function: A large sample twin study. Lung, 189, 323-330.

Klimentidis, Y. C., Vazquez, A. I., de Los Campos, G., Allison, D. B., Dransfield, M. T., \& Thannickal, V. J. (2013). Heritability of pulmonary function estimated from pedigree and whole-genome markers. Frontiers in Genetics, 4, 174.

Li, L., Gao, W., Lv, J., Cao, W., Zhan, S., Yang, H., \& Hu, Y. (2006). Current status of the Chinese national twin registry. Twin Research and Human Genetics, 9, 747-752. 
Li, L., Gao, W., Yu, C., Lv, J., Cao, W., Zhan, S., ... Hu, Y. (2013). The Chinese national twin registry: An update. Twin Research and Human Genetics, 16, 86-90.

McClearn, G. E., Svartengren, M., Pedersen, N. L., Heller, D. A., \& Plomin, R. (1994). Genetic and environmental influences on pulmonary function in aging Swedish twins. Journal of Gerontology, 49, 264-268.

Pang, Z., Ning, F., Unger, J., Johnson, C. A., Wang, S., Guo, Q., ... Lee, L. (2006). The Qingdao twin registry: A focus on chronic disease research. Twin Research and Human Genetics, 9, 758-762.

Rantanen, T., Avlund, K., Suominen, H., Schroll, M., Frandin, K., \& Pertti, E. (2002). Muscle strength as a predictor of onset of ADL dependence in people aged 75 years. Aging Clinical and Experimental Research, 14, 10-15.

Redline, S., Tishler, P. V., Rosner, B., Lewitter, F. I., Vandenburgh, M., Weiss, S. T., \& Speizer, F. E. (1989). Genotypic and phenotypic similarities in pulmonary function among family members of adult monozygotic and dizygotic twins. American Journal of Epidemiology, 129, 827-836.

Reed, T., Fabsitz, R. R., Selby, J. V., \& Carmelli, D. (1991). Genetic influences and grip strength norms in the NHLBI twin study males aged 59-69. Annals of Human Biology, 18, 425432.
Sayer, A. A., Syddall, H., O’Dell, S. D., Chen, X. H., Briggs, P. J., Briggs, R., ... Cooper, C. (2002). Polymorphism of the IGF2 gene, birth weight and grip strength in adult men. Age and Ageing, 31, 468-470.

Tang, W., Kowgier, M., Loth, D. W., Soler Artigas, M., Joubert, B. R., Hodge, E., ... Cassano, P. A. (2014). Large-scale genome-wide association studies and meta-analyses of longitudinal change in adult lung function. PLoS One, 9, e100776.

Tiainen, K., Sipila, S., Alen, M., Heikkinen, E., Kaprio, J., Koskenvuo, M., ... Rantanen, T. (2004). Heritability of maximal isometric muscle strength in older female twins. Journal of Applied Physiology, 96, 173-180.

Tomsey, C. S., Kurtz, M., Kist, F., Hockensmith, M., \& Call, P. (2001). Comparison of PowerPlex 16, PowerPlex1.1/2.1, and ABI AmpfISTR Profiler Plus/COfiler for forensic use. Croatian Medical Journal, 42, 239-243.

Wilk, J. B., Djousse, L., Arnett, D. K., Rich, S. S., Province, M. A., Hunt, S. C., ... Myers, R. H. (2000). Evidence for major genes influencing pulmonary function in the NHLBI family heart study. Genetic Epidemiology, 19, 81-94.

Xu, X., Yang, J., Chen, C., Wang, B., Jin, Y., Fang, Z., ... Weiss, S. T. (1999). Familial aggregation of pulmonary function in a rural Chinese community. American Journal of Respiratory and Critical Care Medicine, 160, 1928-1933. 